\title{
CAD and BIM tools in Teaching of Graphic Representation for Engineering
}

\section{SIGRADI2018 TECHNOPOLITICAS \\ xxii congresso da sociedade iberoamericana de gráfica digital 22th conference of the iberoamerican society of digital graphics 07|08|09|novembro|2018 iau usp | são carlos | sp br}

\author{
Beatriz Campos Fialho \\ Universidade de São Paulo | Brazil | beatriz.fialho@usp.br \\ Heliara A. Costa \\ Universidade Federal do Tocantins, Universidade de São Paulo | Brazil | heliara@uft.edu.br \\ Louise Logsdon \\ Instituto Federal de Mato Grosso, Universidade de São Paulo | Brazil | \\ louise.logsdon@cba.ifmt.edu.br
}

Márcio Minto Fabrício

Universidade de São Paulo | Brazil | marcio@sc.usp.br

\begin{abstract}
BIM technology has represented an advance and a break of the design process' paradigm, impacting both academia and construction market. Reporting a didactic experience in the Civil Engineering graduation, this article aims to understand the teaching and learning process of graphic representation, by using CAD and BIM tools. The research included Literature Review and Empirical Study, whose data collection was based on the application of questionnaires, practical exercises and theoretical test with the students. As a contribution, we highline the complementary nature of the tools and the potentialities of BIM for teaching graphic representation.
\end{abstract}

Keywords: Graphic Representation; CAD System Education; CAE System Education. BIM.

\section{INTRODUÇÃO}

Nas últimas décadas do século $X X$, o aprimoramento das Tecnologias de Informação e Comunicação (TICs) e o desenvolvimento de hardwares para computação gráfica refletiram diretamente no setor da Arquitetura, Engenharia, Construção e Operação (AECO), tornando possível o desenho assistido por computador (CAD - Computer Aided Design) (Aguilar-Molina e Azevedo Junior, 2015).

Lançada em 1970, a tecnologia CAD possibilitou que o processo de representação gráfica, até então inteiramente manual, fosse desenvolvido digitalmente, garantindo melhor qualidade e eficiência. Significou um avanço fundamental por possibilitar a automatização de cálculos complexos e permitir que os projetistas ganhassem mais tempo para tarefas de análise, facilitando também a elaboração e correção de desenhos. Ao longo do tempo, a tecnologia foi sendo aprimorada e tornou-se indispensável na indústria da construção (Costa, Figueiredo e Ribeiro, 2015; Aguilar-Molina e Azevedo Junior, 2015).

O avanço dos computadores, associado à demanda por qualidade e produtividade na indústria da construção, levou ao desenvolvimento de tecnologias que adotassem modelos parametrizados vinculados às informações de projeto e da construção, culminando no que hoje se entende por BIM - Building Information Modeling (Ramos, 2015; Costa, 2017).

O advento da tecnologia BIM tem representado um avanço e uma quebra de paradigma do processo de projeto e de representação gráfica. Isso porque, no BIM, substitui-se a tradicional representação bidimensional dos objetos por um modelo tridimensional inteligente da edificação. $O$ edifício é construído de modo virtual: a concepção do espaço expressa o espaço, e a concepção da forma expressa a forma. Cortes, plantas e fachadas são extraídos automaticamente do modelo da construção, que é uma simulação expressa da edificação (Ruschel, Andrade e Morais, 2013). As alterações e aperfeiçoamentos no modelo são realizados automaticamente nos desenhos 2D (plantas baixas, cortes e elevações), o que facilita o processo de representação gráfica e resulta na melhoria da qualidade da comunicação e do produto final, a construção (Souza, Amorim e Lyrio, 2009).

Diante das vantagens de sua aplicação, o uso de softwares BIM vem sendo incorporados aos processos da produção de edifícios com bastante velocidade nos últimos anos, por meio de incentivos públicos e iniciativas privadas. No Reino Unido, Dinamarca, Finlândia, Noruega e Estados Unidos há iniciativas governamentais que estimulam o uso do BIM há pelo menos dez anos, contando com exigência em projetos públicos, além da publicação de leis e normas técnicas (ABDI, 2017).

No Brasil, um conjunto de medidas vêm sendo tomadas para regular e difundir o uso do BIM, materializadas há pouco mais de cinco de anos.

Coordenados pela Agência Brasileira de Desenvolvimento Industrial $(\mathrm{ABDI})$ e pelo Ministério da Indústria, Comércio Exterior e Serviços (MDIC), além de parceiros, dentre essas medidas constam a produção de bibliotecas de famílias de componentes, como as do Programa Minha Casa Minha Vida (PMCMV) e da Fundação para o 
Desenvolvimento da Educação (FDE); publicação de Normas Técnicas (ABNT 15965-1:2011, 15965-2, 2012, 15965-3, 2014, 15965-7, 2015); criação do Portal BIM; elaboração de Guias aplicáveis ao BIM; instalação do CEBIM para estudos e difusão do conceito e, recentemente, a publicação do decreto $\mathrm{N}^{0}$. 9377, em 17 de maio de 2018, que institui a Estratégia Nacional de Disseminação do Building Information Modelling, que entre outros objetivos, busca criar condições favoráveis para o investimento, público e privado, em BIM (Media Lab, Estadão, 2018).

No setor privado, a ABDI aponta a existência de aproximadamente de 215 mil empresas no segmento da construção civil em todo território brasileiro, mas o uso do BIM entre estas ainda é pequeno. No entanto, as ações governamentais visam que até 2028, pelo menos 50\% destas empresas adotem o BIM (MDIC, 2018).

Para Succar (CBIC, 2018), é notável um amadurecimento do setor em relação ao uso do BIM, principalmente a partir de 2013, tendo sido fundamental a iniciativa governamental de divulgação e estímulo ao seu uso.

Uma das resistências para a adoção do BIM na iniciativa privada brasileira ainda é a ausência de capacitação de profissionais. Essa questão é colocada como uma das dificuldades para o uso da ferramenta entre os profissionais envolvidos na produção de um edifício, impactando principalmente na compatibilização de projetos, ou seja, na análise necessária que se faz de forma sobreposta entre as diferentes disciplinas, como arquitetura, estrutura, instalações, entre outras (CBIC, 2018).

As alterações que o conceito BIM vem promovendo no setor de AECO, aliadas à necessidade de capacitação de profissionais para este novo cenário, fazem refletir sobre a sua incorporação nos métodos de ensino.

Experiências pedagógicas sobre BIM nos cursos de arquitetura e de engenharia civil tem sido objeto de alguns estudos acadêmicos, como o realizado por Barison e Santos (2010), em 2007, junto a instituições internacionais. Os resultados apontaram que: "a maioria das instituições pesquisadas (82\%) ensina ou discute BIM em cursos ou projetos. A minoria (18\%) começou a introduzir BIM no currículo em 2002, outras (27\%) já haviam introduzido BIM antes de 2002 e, ainda, a maior parte delas $(55 \%)$ começou a introduzir BIM a partir de 2007".

De forma semelhante, Checcucci, Pereira e Amorim (2011) identificaram no Brasil alguns trabalhos com a temática "ensino de BIM", entre os anos de 2005 a 2010. Estes trabalhos buscam demonstrar principalmente as vantagens e desvantagens de tecnologias CAD, processos cognitivos de aprendizado de projeto, representação gráfica e geometria por meio dessas tecnologias, barreiras para o seu emprego, entre outros. Por outro lado, também demonstra - de forma indireta que tais tecnologias vêm sendo cada vez mais empregadas em sala de aula. O resultado do estudo desses dos autores foram resumidos na Tabela 1:
Tabela 1: Estudos sobre Ensino de BIM no Brasil - 2005-2010. Fonte: Checcucci, Pereira e Amorim (2011).

\begin{tabular}{|c|c|c|}
\hline Autores & Ano & Descrição \\
\hline $\begin{array}{l}\text { VINCENTE, } \\
\text { C. C. }\end{array}$ & 2006 & $\begin{array}{l}\text { Ensino de projeto arquitetônico com } \\
\text { ferramentas CAD (Desenho Assistido por } \\
\text { Computador), CAAD (Projeto e Desenho } \\
\text { Assistidos por Computador) (sic) e BIM } \\
\text { (Modelagem de Informações da Edificação) }\end{array}$ \\
\hline $\begin{array}{l}\text { ANDRADE, } \\
\text { M. L. V. X. }\end{array}$ & 2007 & $\begin{array}{l}\text { Ensino de Computação Gráfica Digital (sic), } \\
\text { com objetivo de comparar as vantagens e } \\
\text { desvantagens do uso de modeladores } \\
\text { geométricos versus editores de desenho para } \\
\text { o ensino de projeto. }\end{array}$ \\
\hline
\end{tabular}

ANDRADE, 2007 Experiência de ensino, em que se compara M. L. V. X. et as vantagens e desvantagens de utilização al de três ferramentas gráficas: SketchUp, AutoCAD e ArchiCAD, em fases diferentes da projetação arquitetônica.

FLORIO, W. 2007 Examina as contribuições da BIM nas ações cognitivas realizadas pelo projetista durante 0 processo de criação e de desenvolvimento de projeto de arquitetura.

NARDELLI, 2008 Aponta alguns desafios sobre a utilização de E. BIM e seu reflexo nas escolas de arquitetura

RUSCHEL, R. 2008 Experiência de ensino que visa abordar C.; $\quad$ barreiras de cunho cultural para a adoção do GUIMARÃES, CAD 4D.

F. A. B.

ARAÚJO, N. 2009 Apresenta a importância da experimentação S. no ensino de projeto de arquitetura. Mostra que a tecnologia facilitou as simulações e investigações projetuais, proporcionando uma melhor compreensão e tomada de decisão nos projetos.

OLIVEIRA, M. 2009 Questiona a maneira de ensinar a geometria de L. projetiva nos cursos de arquitetura e urbanismo diante das mudanças no conhecimento e na representação da forma, estabelecida pela gráfica digital com as tecnologias CAD e BIM.

MENEZES, A. 2010 Mostram as mudanças metodológicas no processo de projeto decorrentes da implantação da tecnologia BIM. Fazem um comparativo sobre o processo linear de ensino e de prática de projeto, demostrando que a tecnologia BIM implica numa nova abordagem para a prática profissional, com consequências para o ensino.

Ruschel, Andrade e Morais (2013), em pesquisa sobre experiências de ensino de BIM, nacionais e internacionais, propuseram uma classificação dos "Estágios de adoção de BIM e níveis de competência”. A partir dessa classificação, concluem que a maioria dos cursos no Brasil aborda o ensino apenas em disciplinas isoladas, com algumas iniciativas que visam integrar o ensino de BIM às disciplinas de projeto estrutural e arquitetônico. 
Souza e Ulbrich (2013) discutem a importância de conciliar conhecimentos de desenho técnico e de ferramentas tecnológicas na definição de métodos de ensino de engenharia. A fim de auxiliar os estudantes e professores de engenharia na aplicação e uso dos sistemas computacionais, sugerem que as disciplinas sejam organizadas em uma sequência de três fases: 1. "Desenho técnico" à mão livre, com enfoque no "raciocínio tridimensional e na interpretação de desenhos de engenharia"; 2. "Desenho técnico auxiliado por computador", voltado para o aprofundamento dos "conceitos de desenho técnico" por meio de ferramentas CAD 2D; 3. "Engenharia integrada por computador", destinada à aplicação de "conceitos teóricos e práticos sobre tecnologias, integração de sistemas CAx (CAD/CAM/CAE/CAl, etc.) e tecnologias avançadas de fabricação".

No curso de engenharia civil da Escola de Engenharia da USP de São Carlos, o BIM vem sendo introduzido pontualmente em algumas disciplinas. Na disciplina de Desenho, que é oferecida no primeiro semestre do curso e contempla o conteúdo de representação gráfica, a nova ementa prevê o uso de softwares CAD e BIM - AutoCad e Revit.

Este artigo parte do relato de experiência dos autores, que acompanharam a disciplina de Desenho no primeiro semestre de 2018. O objetivo é compreender o processo de ensino e aprendizagem de desenho, por meio da aplicação de ferramentas CAD e BIM. Mais especificamente, busca-se comparar a qualidade da representação gráfica dos exercícios produzidos em cada um dos softwares (AutoCad e Revit), analisando também a percepção dos estudantes quanto ao uso das ferramentas.

A relevância do trabalho está em discutir a incorporação de ferramentas digitais inovadoras em disciplinas de representação gráfica, contribuindo para a formação do profissional de engenharia.

\section{MÉTODO}

Para alcançar os objetivos propostos, trabalho foi estruturado em quatro etapas (Figura 1), com as seguintes técnicas de coleta de dados:

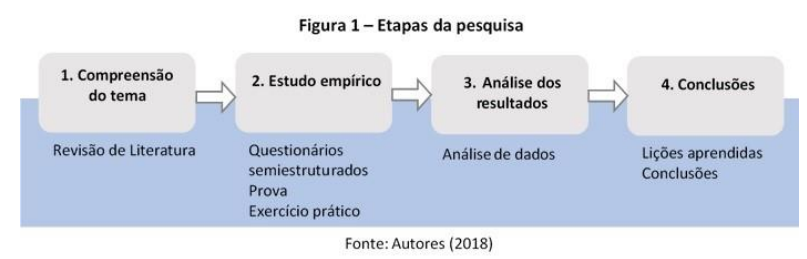

Figura 1: Etapas da pesquisa. Fonte: Os autores.

Para a compreensão do tema, foi realizada uma revisão de literatura sobre $o$ ensino e aprendizagem de ferramentas digitais para representação gráfica. Parte desta revisão está apresentada na Introdução deste artigo.

O estudo empírico dividiu-se ainda em cinco etapas, cada uma visando a obtenção de dados específicos, valendo-se, para isso, de técnicas distintas.

1. Caracterização da disciplina: análise da ementa e do plano da disciplina.
2. Identificação do perfil da turma: verificação junto aos estudantes dos conhecimentos prévios sobre representação gráfica e/ou sobre as ferramentas digitais investigadas, por meio de questionário semiestruturado aplicado digitalmente com suporte da ferramenta Google Forms (Questionário 1) [1];

3. Avaliação do desempenho por meio da aplicação de prova teórica sobre representação gráfica.

4. Avaliação do desempenho dos estudantes na elaboração de exercícios práticos de desenho arquitetônico de uma residência unifamiliar, inicialmente com o uso do software AutoCAD e posteriormente, do software Revit, ambos da empresa Autodesk.

5. Análise da opinião dos estudantes sobre a operação das ferramentas na produção dos desenhos, suas vantagens e desvantagens, por meio da aplicação de questionários semiestruturados digitais (Questionários 1, 2 e 3).

Após a coleta dos dados, deu-se início à análise dos resultados: questionários, prova e exercícios foram analisados, a fim de avaliar o aprendizado de representação gráfica e o conhecimento operacional das ferramentas empregadas.

Por fim, na última etapa foram apresentadas as conclusões e as lições aprendidas com esta pesquisa.

\section{RESULTADOS}

\section{A DISCIPLINA}

De caráter introdutório, a disciplina de "Desenho" visa abordar conhecimentos teóricos e práticos de representação gráfica, empregados na produção de desenhos técnicos e arquitetônicos, a serem aprimorados ao longo do curso de engenharia civil. A carga horária total é de $120 \mathrm{~h}$, distribuídas em $80 \mathrm{~h}$ de aulas presenciais, com duração de $4 \mathrm{~h}$ cada, e $40 \mathrm{~h}$ de trabalho extraclasse. As aulas foram ministradas em laboratório de informática, com acesso aos softwares AutoCad 2018 e Revit 2018, ambos da empresa Autodesk. Essas ferramentas atendem aos propósitos da disciplina e possuem versão educacional gratuita, o que viabiliza a utilização pelos estudantes dentro e fora de sala.

A definição dos métodos de ensino-aprendizagem buscou diversificar as formas de construção do conhecimento, a fim de engajar os estudantes e atender a distintos estilos de aprendizagem (Wankat e Oreovicz, 2015). Os métodos compreenderam aulas expositivas a respeito de normas técnicas e convenções aplicáveis ao desenho, bem como aulas práticas, com aplicação de comandos básicos das ferramentas AutoCAD e Revit. Essas aulas buscaram ainda demonstrar a sequência lógica do processo de desenho, partindo da representação dos elementos construtivos até a diagramação de pranchas para a plotagem.

Além das aulas formais, a disciplina reservou parte da carga horária para atividades extraclasse, a fim de viabilizar a dedicação à investigação teórica e ao desenvolvimento prático. Como referências bibliográficas, foram disponibilizados livros, apostilas, teses e normas técnicas (ABNT NBR 6492:1992; ABNT NBR 10068:1987; ABNT NBR 10126:1987; ABNT NBR 13142:1999). No sentido de orientar a prática autônoma do desenho, foram indicados para consulta vídeos e tutoriais específicos sobre o uso das ferramentas AutoCAD e Revit. 
Em coerência com a abordagem de ensino-aprendizagem, a disciplina contemplou quatro avaliações distribuídas ao longo do período, cuja soma resultou na nota final: uma prova individual teórica, abordando aspectos normativos e prescritivos de representação gráfica, com peso $25 \%$; três exercícios práticos em dupla, com enfoque na produção de desenhos arquitetônicos utilizando as ferramentas digitais de desenho, com peso $75 \%$ cada um.

Os Exercícios Práticos 1 e 2 foram realizados a partir do levantamento in loco de uma residência unifamiliar escolhida pelos estudantes, representada inicialmente no AutoCAD e posteriormente no Revit. Para ambos, foram fornecidos templates baseados nas normas pertinentes com configurações de elementos como textos, linhas, pranchas, a fim de padronizar a apresentação dos trabalhos. Dessa forma, foi possível avaliar a qualidade técnica da representação do mesmo objeto arquitetônico. O Exercício Prático 3 propôs a modelagem BIM de um projeto arquitetônico pré-definido pelo professor, a fim de consolidar as habilidades na operação de comandos básicos do Revit. Esta avaliação não integrou os resultados desta pesquisa, devido ao fato de se aprofundar em apenas uma ferramenta.

Como afirmam Wankat e Oreovicz (2015), a aplicação de avaliações em maior quantidade e diversidade é benéfica para o aprendizado, reduzindo a influência de cada avaliação na nota final e criando uma rotina de estudos. A correção da prova e dos exercícios práticos foi realizada a partir de critérios objetivos, fornecendo aos estudantes um feedback dos aspectos a serem aprimorados e a oportunidade de revisão para uma nova correção. Os resultados das avaliações subsidiaram a discussão proposta neste artigo.

A plataforma Google Classroom $^{1}$ foi utilizada como sistema de gerenciamento de conteúdo, auxiliando no fornecimento de materiais didáticos, na distribuição e avaliação de exercícios e na comunicação entre professor e estudantes.

\section{PERFIL DA TURMA}

A caracterização da turma foi realizada a partir do Questionário 1, respondido por 26 estudantes em 17 de maio de 2018. Os dados apontam um perfil homogêneo, formado por estudantes do primeiro período do curso de engenharia civil e sem experiência profissional na área da construção civil. Desses, apenas quatro $(15,4 \%)$ possuíam conhecimentos prévios sobre representação gráfica, adquiridos de diferentes formas - curso de aprendizagem industrial em mecânica de usinagem, curso de desenho arquitetônico e aulas do ensino técnico -, dos quais dois (8\%) possuíam formação de Técnico em Edificações. Quantos ao uso dos softwares de projeto de Arquitetura e Engenharia, cinco estudantes (20\%) haviam utilizado o AutoCAD, sendo que um deles dominava o SketchUp.

O perfil da turma reforça o caráter introdutório da disciplina e a importância de trabalhar conteúdos teóricos e práticos básicos de representação gráfica, bem como comandos dos softwares empregados.

\footnotetext{
${ }^{1}$ Disponível em: <https://classroom.google.com>. Acesso em 20
} fev. 2018.

\section{DESEMPENHO NA PROVA TEÓRICA}

A prova individual foi aplicada para 29 estudantes em 19 de abril de 2018, com o propósito de avaliar os conhecimentos teóricos sobre geometria descritiva, vistas ortográficas, perspectivas e desenho técnico, abordados no início da disciplina. A prova contemplou oito questões de múltipla escolha, com alternativa única e com múltiplas alternativas, totalizando 10,0 pontos. A média aritmética das notas foi de 7,0 pontos, considerada satisfatória para a disciplina (Gráfico 1). Os estudantes apresentaram maior dificuldade em questões relativas à representação de projetos de arquitetura, que seriam posteriormente reafirmadas nos exercícios práticos.

Gráfico 1: Distribuição das notas da prova por estudante e média das notas. Fonte: Os autores.

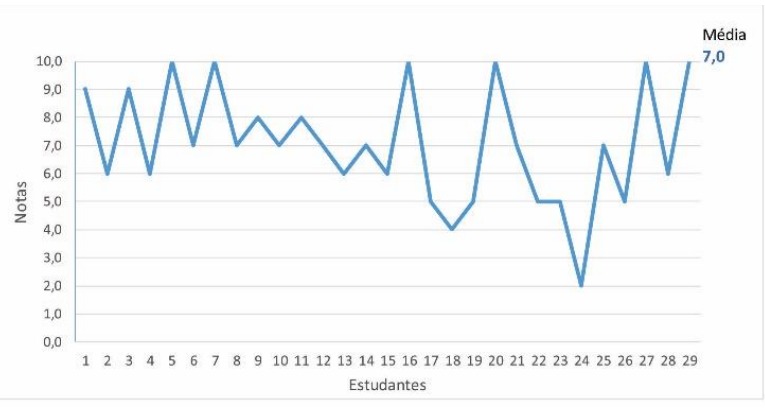

\section{DESEMPENHO NOS EXERCÍCIOS PRÁTICOS}

Os Exercícios Práticos 1 e 2 foram entregues, respectivamente, nos dias 10 de maio de 2018 e 21 de junho de 2018. Como produtos, foram apresentadas pranchas impressas em formato $A 1$, contendo Planta Baixa, Corte Transversal, Corte Longitudinal, Fachada Frontal e Planta de Cobertura da edificação. Para a avaliação, foi estruturado um roteiro comum de verificação do projeto arquitetônico, com critérios relativos ao conteúdo e à qualidade gráfica dos desenhos e pranchas, conforme previsto nas normas técnicas pertinentes. Os itens foram avaliados em uma escala de 0 a 1,0 , sendo 0 = insatisfatório, $0,5=$ parcialmente satisfatório e 1,0 = satisfatório. No Exercício Prático 1, elaborado com o uso do AutoCAD, a média das notas foi de 7,4/10,0 pontos, enquanto no Exercício Prático 2, desenvolvido no Revit, a média de notas foi de 8,3/10,0 pontos (Gráfico 2).

Gráfico 2: Distribuição das notas dos Exercícios Práticos 1 e 2 por dupla e média das notas. Fonte: Os autores.

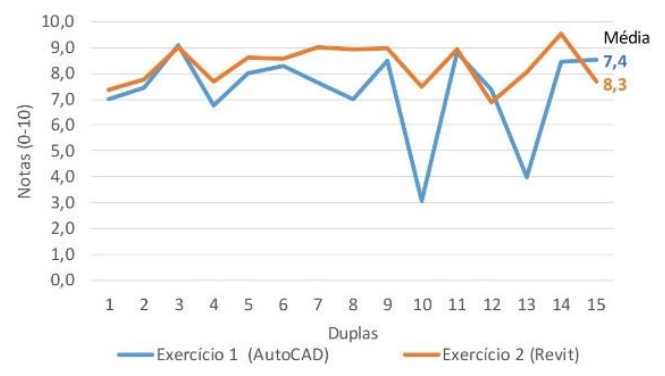

$\mathrm{Na}$ análise das pranchas e desenhos do Exercício Prático 1 , as principais deficiências foram identificadas na representação dos elementos construtivos (paredes, esquadrias, etc.), na hierarquia de linhas, inserção de textos, cotas e símbolos (norte, níveis) e na diagramação 
e apresentação das pranchas. Ressalta-se que, para a maior parte da turma, este foi o primeiro contato com o desenho arquitetônico e com a ferramenta.

Gráfico 3: Distribuição das notas dos conteúdos dos Exercícios Práticos 1 e 2 por dupla e média das notas. Fonte: Os autores.
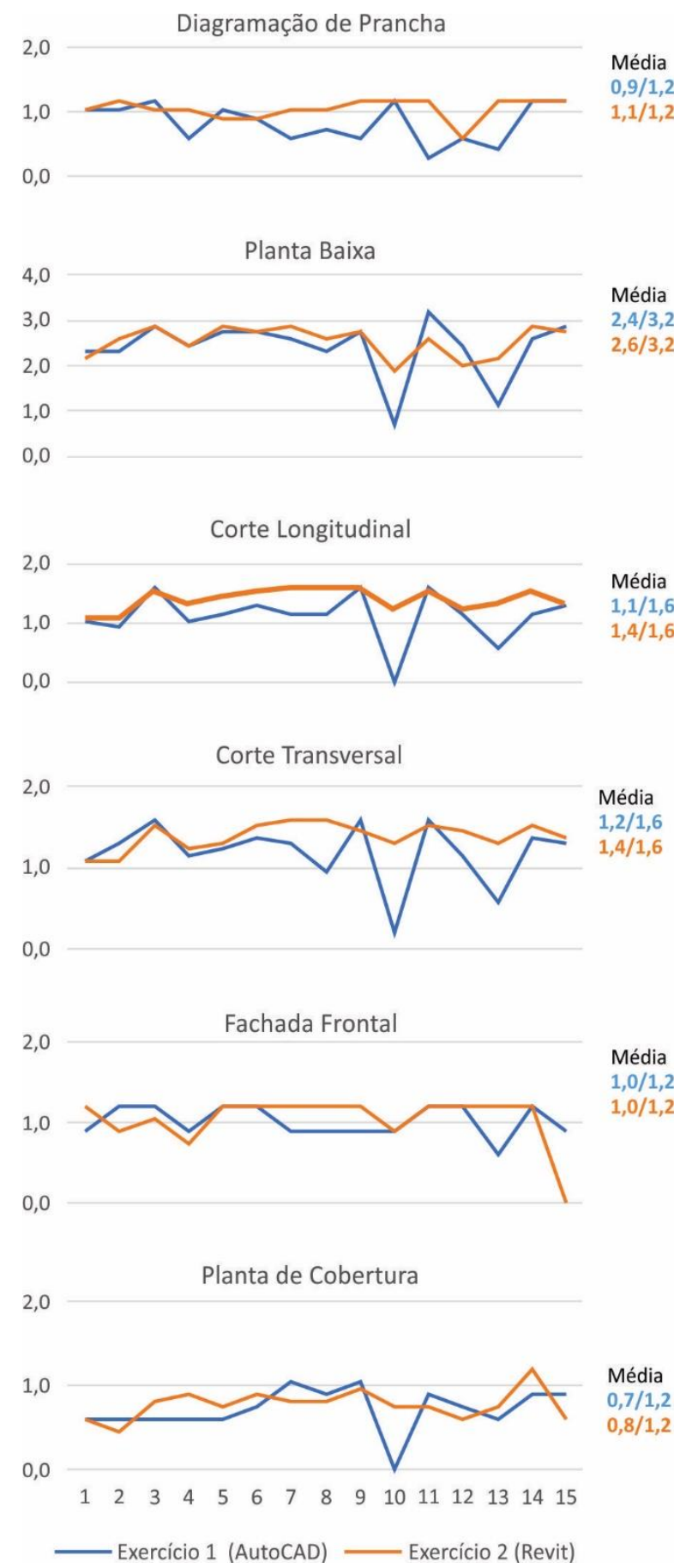

Como ilustra o Gráfico 3, no Exercício Prático 2 houve uma melhoria da qualidade da diagramação de pranchas e na produção dos desenhos técnicos, especialmente na representação gráfica de cortes que são extraídos de forma automática do modelo virtual da edificação. A qualidade da fachada frontal se manteve constante. Foram verificadas falhas na caracterização dos elementos construtivos, na inserção de textos e símbolos, mas em menor número do que no exercício anterior.
O Revit automatiza diversas funções, como o cálculo de áreas, a geração de vistas e a extração de tabelas, facilitando a produção dos documentos do projeto. $O$ processo de notação dos desenhos (especificação de elementos, identificação de esquadrias, indicação de níveis) também são parametrizados, mas exige que o aluno insira estes elementos em cada planta, ou seja, não basta inserir em uma delas que, automaticamente, estarão presentes nas outras. Isso pode justificar as falhas recorrentes em ambos exercícios. Acredita-se também que tais deficiências tenham ocorrido por uma necessidade de familiarização com a linguagem técnica e com os elementos construtivos das edificações, aspectos que podem ser aprofundados ao longo do curso em outras disciplinas.

\section{A PERCEPÇÃO DOS ESTUDANTES}

Para avaliar a percepção dos estudantes sobre a operação das ferramentas digitais e o aprendizado de representação gráfica, foram aplicados três questionários. O Questionário $1^{2}(17 / 05 / 2018)$, também utilizado para caracterização da turma, foi aplicado após a entrega do Exercício Prático 1 e continha questões relacionadas ao uso do AutoCAD. Seguindo o mesmo padrão do anterior, o Questionário $2^{3}$ foi realizado em 21 de junho de 2018 e apresentou questões sobre a produção de desenhos no Revit. Na mesma data, foi aplicado o Questionário $3^{4}$, abordando questões comparativas entre as duas ferramentas. Os questionários contemplaram três tipos de questões: resposta simples descritiva; múltipla escolha; e escala linear, variando de 1 a 5, sendo 1 = muito difícil, 2 = difícil, 3 = neutro, 4 fácil e 5 muito fácil.

\section{QUESTIONÁRIO 1 - AUTOCAD}

Consultados sobre a leitura geral da interface e a operação dos comandos no AutoCAD, 13 estudantes (50\%) a consideraram fácil, enquanto nove (34\%) a avaliaram como neutra. Quanto à produção de desenhos técnicos (planta baixa, cortes, planta de cobertura e fachadas), a maior parte da turma considerou que o uso da ferramenta é difícil ouneutra (nem fácil, nem difícil), sendo que poucos consideram a ferramenta de uso "fácil" para estas funções.

Grande parte dos estudantes avaliou o AutoCAD como "fácil" ou "muito fácil" nas operações de inserção de texto (11 estudantes, 42\%), cotas (17 estudantes, 65\%), símbolos (15 estudantes, 57\%) e hachuras (15 estudantes, $57 \%$ ). Nas configurações de escala e linhas, a maior parte da turma teve opinião neutra ou negativa ("difícil" e "muito difícil") quanto a sua operação. A montagem de pranchas foi considerada "fácil" ou "muito fácil" para 11 estudantes (42\%) e "difícil" ou "muito difícil" para 4 estudantes (15\%).

Em síntese, cerca de 53\% dos entrevistados considerou o AutoCAD adequado para o aprendizado de representação gráfica. Dentre os estudantes que fizeram comentários adicionais, a maioria achou a ferramenta interessante, e demonstrou motivação em se aprofundar nos estudos para aprimorar a qualidade dos desenhos. Alguns

\footnotetext{
2 https://goo.gl/forms/HZLPGqo9UPAPDZOs 1

${ }^{3} \mathrm{https}: / /$ goo.gl/forms/YAGHdIQFQyXmXF1S2

${ }^{4} \mathrm{https} / / / g 00 . g l / f o r m s /$ OOAGSjkjEqQoibi2
} 
destacaram, no entanto, a necessidade de aumentar o número de aulas práticas, dada a robustez do software.

Gráfico 4: Percepção dos estudantes quanto ao uso do AutoCAD. Fonte: Os autores.

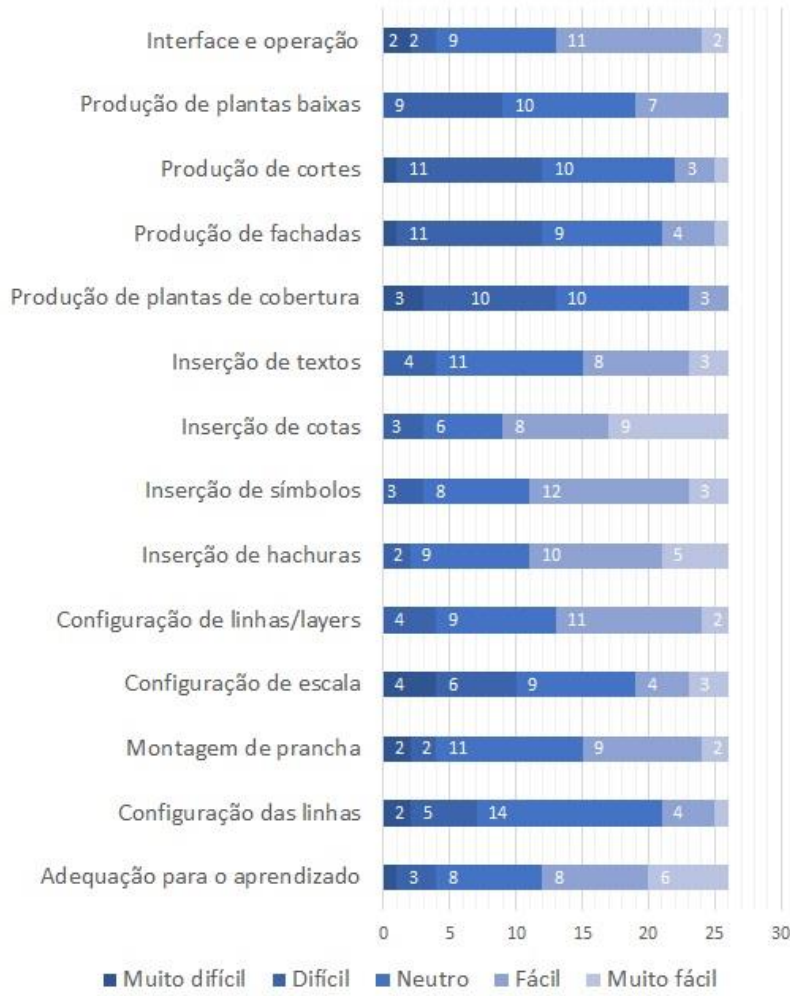

\section{QUESTIONÁRIO 2 - REVIT}

25 estudantes responderam às questões do Questionário 2 que faziam referência ao uso do Revit. Boa parte da turma (14 estudantes, 56\%) considerou "fácil" ou "muito fácil" a leitura geral da interface e a operação de comandos no software. Da mesma forma, a grande maioria avaliou positivamente a facilidade de elaborar os desenhos técnicos: planta baixa (20 estudantes, $80 \%$ ), cortes (23 estudantes, 92\%), fachadas (20 estudantes, $80 \%$ ) e plantas de cobertura (18 estudantes, $72 \%$ ).

Em todas as questões seguintes, predominaram respostas positivas: a maior parte dos estudantes avaliou como "fácil" ou "muito fácil" a inserção de textos (16 estudantes, $64 \%$ ) e cotas (18 estudantes, 72\%), bem como a configuração de escalas (14 estudantes, 56\%) e a montagem de pranchas (15 estudantes, 60\%). Houve uma quantidade maior de respostas "difícil" ou "muito difícil" na inserção de símbolos ( 6 estudantes, 24\%), hachuras (8 estudantes, 31\%) e na configuração de penas (6 estudantes, 24\%).

Por fim, cerca de $72 \%$ dos entrevistados considerou o Revit adequado para o aprendizado de representação gráfica. Dentre os estudantes que fizeram comentários adicionais, alguns mencionaram que o software é bem "dinâmico e poderoso", por simular a construção da edificação em três dimensões. Destacam, no entanto, a dificuldade em alterar espessuras e cores das linhas, o que é mais simples no AutoCAD.
Gráfico 5: Percepção dos estudantes quanto ao uso do Revit. Fonte: Os autores.

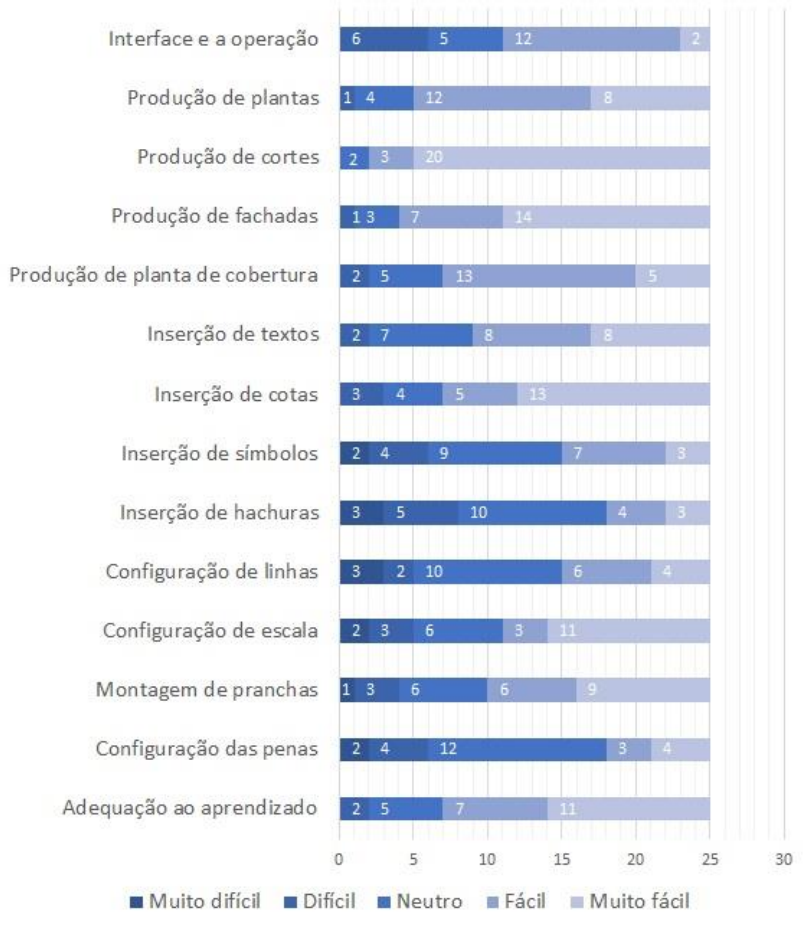

\section{QUESTIONÁRIO 3 - AUTOCAD X REVIT}

21 estudantes responderam às questões do Questionário 3 que buscavam uma análise comparativa entre a operação do AutoCAD e do Revit. A maioria considerou que o Revit é melhor que o AutoCAD para a produção dos desenhos técnicos: plantas baixas (13 estudantes, 62\%), cortes (20 estudantes, 95\%), elevações (20 estudantes, $95 \%$ ) e plantas de coberturas (16 estudantes, 76\%). E continuou seguindo como o preferido em quase todos os itens levantados: inserção de cotas, configuração de escalas, montagem de prancha e plotagem.

Em alguns itens específicos o AutoCAD foi considerado melhor que o Revit: inserção de textos (11 estudantes, $52 \%$ ), símbolos (13 estudantes, 62\%) e configuração de linhas (11 estudantes, 52\%).

Por fim, $80 \%$ da turma (17 estudantes) considera que o Revit demanda menor tempo na produção de desenhos técnicos e na montagem das pranchas, e 76\% (16 estudantes) considera que o Revit é o software que melhor contribui para o aprendizado de representação gráfica. Muitos justificaram o porquê: O Revit é mais prático e permite visualizar melhor a edificação. No entanto, ressaltaram a dificuldade de configurar linhas e acrescentar informações em texto, que, segundo os estudantes, é mais fácil no AutoCAD. 
Gráfico 6: Comparativo entre o uso do AutoCAD e do Revit, segundo a percepção dos estudantes. Fonte: Os autores.

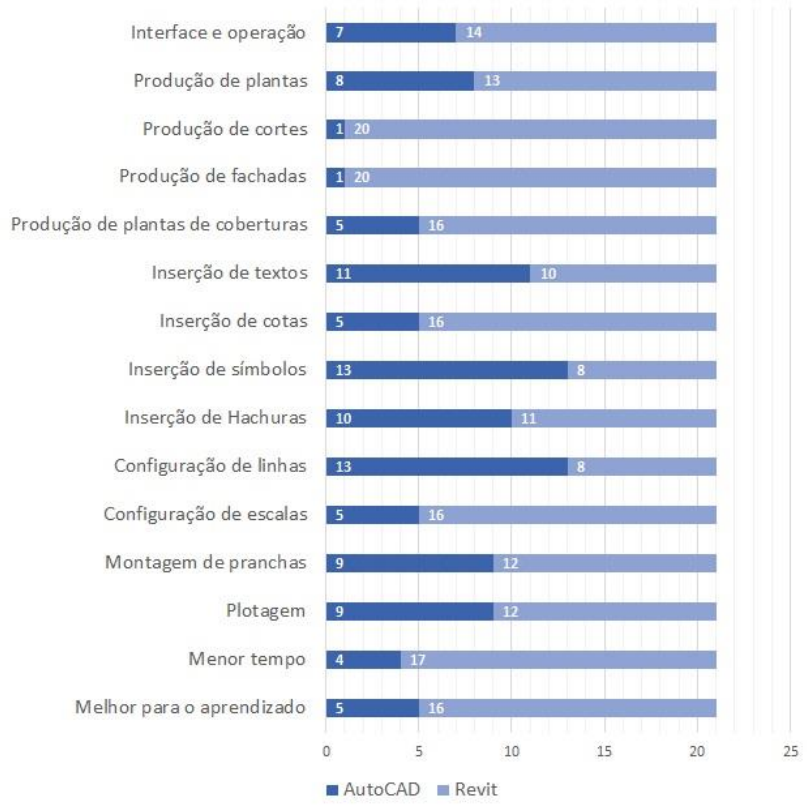

\section{DISCUSSÃO DOS RESULTADOS}

Em atendimento aos métodos de ensino, a disciplina contemplou fundamentos teóricos de representação gráfica e sua aplicação prática por meio dos softwares AutoCAD e Revit, conforme sequência lógica prevista por Souza e Ulbrich (2013). Embora não seja diretamente integrada a outros cursos, como projeto de estruturas, por exemplo, a disciplina introduz importantes conceitos sobre a representação, visualização e construção de objetos na construção civil, contribuindo para o aprendizado futuro em outras disciplinas.

Acredita-se que alguns fatores colaboraram para os bons resultados alcançados na disciplina, como a disponibilidade de carga horária extra para o desenvolvimento autônomo de desenhos, o fornecimento de materiais didáticos complementares (vídeos, tutoriais) e a distribuição das avaliações ao longo do período, demandando dos estudantes o estudo constante do tema e dos softwares. Além disso, o sistema online de gerenciamento de conteúdo auxiliou na troca de informações com a turma, que ocorreu de forma dinâmica e eficiente.

Importante ressaltar que $15 \%$ da turma (4 estudantes) possuía conhecimento prévio de representação gráfica e sabia utilizar o AutoCAD. Dois deles, inclusive, tinham formação de Técnico em Edificações. Para estes, a primeira parte da disciplina foi de fácil processamento, o que refletiu no bom desempenho dos mesmos no primeiro exercício. No entanto, os estudantes que não tinham conhecimento prévio também tiveram desempenho satisfatório nos exercícios, apresentando algumas deficiências que devem ser supridas naturalmente, com a prática.

Analisando os desenhos entregues pelos estudantes, foi possível observar uma evolução da qualidade da representação gráfica: os desenhos elaborados em Revit (Exercício 2) apresentaram melhor qualidade gráfica que os elaborados em AutoCAD (Exercício 1). Este fato foi refletido nas notas da turma, que alcançou maior média no Exercício 2 (Gráfico 2). Isso pode ser justificado pela robustez do Revit, que facilita, por exemplo, a extração de cortes e elevações, mas também porque os estudantes já teriam consolidado melhor os conceitos de representação gráfica através das avaliações anteriores da disciplina (prova teórica e exercício 1 - AutoCAD).

De uma maneira geral, o Revit se mostrou como o software preferido pelos estudantes para o aprendizado de representação gráfica. Mencionaram que o mesmo é mais fácil para a elaboração de plantas, cortes e elevações, sendo considerado mais "rápido", "prático" e "didático", pelo fato de a edificação ser modelada em 3D, o que facilitaria 0 entendimento do processo, bem como a extração dos desenhos em 2 dimensões. Apenas 5 estudantes preferem o AutoCAD - provavelmente aqueles que possuíam um domínio prévio neste software. A justificativa é que o Revit é muito "engessado", como afirma este estudante:

\begin{abstract}
"Com o AutoCAD, você pode representar o que quiser no desenho: se necessitar fazer determinado objeto, você consegue fazer a partir das linhas. No Revit, é necessário procurar alguma coisa que você queira representar" [Resposta de estudante].
\end{abstract}

Cabe mencionar a opinião de um outro estudante, com relação às ferramentas e ao ensino de representação gráfica. Sua fala ressalta a importância do AutoCAD como prancheta digital, que mantém a forma tradicional de produzir os desenhos:

\begin{abstract}
"O Revit possui uma melhor visualização, entretanto para um aprendizado inicial de representação gráfica e noções sobre as vistas o Autocad é melhor, uma vez que no Revit já vem pronto" [Resposta de estudante].
\end{abstract}

De fato, o BIM apresenta uma mudança de paradigma na forma de pensar a representação gráfica e, por esta razão, sua implantação nos cursos de graduação tem enfrentado muitos desafios.

\section{CONSIDERAÇÕES FINAIS}

Este artigo foi baseado em uma experiência pontual de adoção do BIM no curso de engenharia civil da Escola de Engenharia de São Carlos (EESC-USP), dentro da disciplina de Desenho. No entanto, Checcucci, Pereira e Amorim (2013) citam diversos autores ${ }^{5}$ que recomendam a implementação integrada do BIM em diferentes disciplinas da graduação, e através de práticas colaborativas. É um desafio para a instituição, por sugerir que se alterem culturas estabelecidas, rotinas e práticas de trabalho consolidadas. Por outro lado, acreditam que a adoção integrada do BIM está mais alinhada com as demandas do mercado de trabalho, por simular situações práticas em que 0 estudante pode testar seus conhecimentos.

Sendo assim, a experiência relatada neste artigo levanta apenas uma das potencialidades da tecnologia, mas

\footnotetext{
${ }^{5}$ Rebolj, Menzel e Dinevski (2008); Sacks e Barak (2010); Wong,
} Wong e Nadeem (2011). 
experiências mais interdisciplinares precisam ser incluídas no curso de engenharia da EESC-USP, para a exploração de todo o potencial do BIM e para a melhor preparação dos estudantes para o mercado de trabalho. Cabe à Instituição, no entanto, uma análise criteriosa de seus objetivos e condições para tal.

Por outro lado, a experiência de trabalhar CAD e BIM em uma mesma disciplina foi considerada positiva, justamente por encontrarmo-nos em um momento de transição entre estas tecnologias. Ainda hoje, algumas disciplinas do curso de Engenharia utilizam ferramentas CAD em seus métodos de ensino. Da mesma forma, diversas empresas e instituições públicas têm seus processos de projeto baseados em tecnologias CAD 2D, o que demanda dos profissionais de engenharia a capacidade de empregá-las adequadamente, seja na produção de projetos, na gestão da construção ou na interface com outras disciplinas.

Assim, acredita-se que os softwares trabalhados na disciplina são complementares para a construção do conhecimento de representação gráfica, pois o AutoCAD, enquanto "prancheta digital", reforça os conteúdos básicos de desenho, a partir do qual foi criado, e o Revit propõe uma visão de modelagem do edifício, centrada da "construção virtual" do objeto.

A experiência em tratar de representação gráfica com os dois softwares foi bastante interessante e possivelmente será continuada, com as próximas turmas. Serão buscados meios de suprir as deficiências encontradas, em vias de aproveitar melhor o tempo da disciplina com as atividades práticas. Monitorias e atividades extraclasses serão bem-vindas, assim como a avaliação da disciplina por parte dos estudantes, para que haja uma retroalimentação do processo de ensino.

\section{AGRADECIMENTOS}

Agradecemos aos estudantes da disciplina de Desenho do curso de engenharia civil da EESC-USP (2018/1), pela fundamental contribuição a esta pesquisa. Ao Instituto Federal de Mato Grosso (IFMT), pelo afastamento de doutorado concedido a um dos autores. Por fim, ao Conselho Nacional de Desenvolvimento Científico e Tecnológico (CNPq), pela bolsa produtividade fornecida ao orientador do trabalho.

\section{REFERÊNCIAS}

Agência Brasileira de Desenvolvimento Industrial. (2017). Mobilização para Difusão do BIM no Brasil Iniciativas da ABDI, MDIC e parceiros. Oficina sobre desenvolvimento de estratégia de implantação do BIM no Complexo Industrial da Saúde. Brasília, DF. Retrieved from: http://portalarquivos2.saude.gov.br/images/pdf/2017/setembro /04/2.pdf.

Aguilar-Molina, M. L., \& Azevedo Junior, W. (2015). O ensino/aprendizado do BIM no curso de Engenharia Civil da UFJF. In VII Encontro de Tecnologia de Informação e Comunicação na Construção. Recife, PE.

Andrade, M. L. V. X., \& Ruschel, R. C. (2011). Building Information Modeling (BIM). In D. C. C. K. Kowaltowski, D. de C. Moreira, J. R. D. Petreche, \& M. M. Fabricio (Eds.), O processo de projeto em arquitetura. São Paulo, SP: Oficina de Textos.
Barison, M. B.; Santos, E. T. (2010). Estratégias de Ensino BIM: uma visão geral das abordagens atuais. In: INTERNATIONAL CONFERENCE ON COMPUTING IN CIVIL AND BUILDING ENGINEERING, 12., Nottingham, 2010. Proceedings... Nottingham: ICCCBE, 2010. Retrieved from: <http://www.uel.br/pessoal/barison/Artigos_Tese/p288p.pdf>.

Câmara Brasileira da Indústria da Construção. (2018). BIM: inovação e tecnologia modernizam indústria da construção. São Paulo. Retrieved from: <https://cbic.org.br/wpcontent/uploads/2018/03/ESPECIAL-BIM-CBICEstada\%CC\%830.pdf>

Checcucci, E. S., Pereira, A. P., \& Amorim, A. L. (2011). A difusão das tecnologias BIM por pesquisadores do Brasil. In: TIC 2011 - V Encontro de Tecnologias de Informação e comunicação na construção. Salvador, BA, V, 2011, Salvador

Checcucci, E. S., Pereira, A. P., \& Amorim, A. L. (2013). "Modelagem Da Informação Da Construção (BIM) No Ensino de Arquitetura." In Proceedings of the XVII Conference of the Iberoamerican Society of Digital Graphics - SIGraDi: Knowledge-Based Design, 307-311.

Costa, G. C. L. R., Figueiredo, S. H., \& Ribeiro, S. E. C. (2015). Estudo Comparativo da Tecnologia CAD com a Tecnologia BIM. Revista de Ensino de Engenharia, 34(2), 11-18. https://doi.org/10.15552/2236-0158/abenge.v34n2p11-18

Costa, J. D. S. (2017). Modelagem geométrica como mediadora da construção do conhecimento em Desenho Técnico no Curso de Edificações do IFBA, Campus Salvador. Dissertação de mestrado, Universidade Estadual de Feira de Santana, Feira de Santana, BA, Brasil.

Ministério da Indústria, Comércio Exterior e Serviços. (2018). Governo federal lança estratégia para promover inovação na indústria da construção. Retrieved from: http://www.mdic.gov.br/index.php/component/content/article?i $\mathrm{d}=3296$.

MEDIA LAB Estadão. (2018). Especial Tecnologia na Construção. São Paulo. Suplemento Especial, Estadão, CBIC, SENAI, 2018. 8 pag.

Ramos, S. M. E. (2015). Representação gráfica em Projetos de Arquitetura: Um novo Paradigma. In XI Seminário do Programa de Pós-Graduação em Desenho, Cultura e Interatividade. Feira de Santana, BA.

Rebolj, D., Menzel, K. \& Dinevski, D. (2008). A Virtual Classroom for information Technology in Construction. Computer Applications in Engineering Education. 16(2), p. 105-114.

Ruschel, R. C. Andrade, M. L. V. X; Morais, M. (2013). O ensino de BIM no Brasil: onde estamos? Ambient. constr., Porto Alegre, v. 13, n. 2, p. 151-165, June 2013. http://dz.doi.org/10.1590/S1678-86212013000200012.

Sacks, R.; Barak, R. (2010). Teaching Building Information Modeling as an Integral Part of Freshman Year Civil Engineering Education. Journal of professional issues in engineering education and practice. 136(1), p. 30-38.

Souza, A. F.; Ulbrich, C. B. L (2013). Engenharia integrada por computador e sistemas CAD/CAM/CNC: princípios e aplicações. 2ª ed. São Paulo: Artliber. 358 p.

Souza, L. L. A., Amorim, S. R. L., \& Lyrio, A. M. (2009). Impactos do uso do BIM em escritórios de arquitetura: Oportunidades no mercado imobiliário. Gestão E Tecnologia de Projetos, 4(2), 27-53. https://doi.org/10.4237/gtp.v4i2.100.

Wankat, P. C.; Oreovicz, F. S (2015). Teaching Engineering. Purdue University Press.

Wong, K. A.; Wong, K. F.; Nadeem, A. (2011). Building Information Modeling for tertiary construction education in Hong Kong. Journal of Information Technology in $\begin{array}{llll}\text { Construction } & \text { (ITCon). } & 16, & \text { p. }\end{array}$ 